\title{
miR-128-3p suppresses hepatocellular carcinoma proliferation by regulating PIK3R1 and is correlated with the prognosis of $\mathrm{HCC}$ patients
}

\author{
CHAO-YUAN HUANG ${ }^{*}$, XIN-PING HUANG ${ }^{*}$, JI-YE ZHU, ZHI-GANG CHEN, XIAN-JIAN LI, \\ XUE-HUI ZHANG, SHAN HUANG, JIAN-BO HE, FANG LIAN, YIN-NONG ZHAO and GUO-BIN WU \\ Department of Hepatobiliary Surgery, Affiliated Tumor Hospital of Guangxi Medical University, \\ Nanning, Guangxi 530021, P.R. China
}

Received March 9, 2015; Accepted April 15, 2015

DOI: $10.3892 /$ or.2015.3936

\begin{abstract}
RNAs (miRNAs) are known to be involved in the pathogenesis of hepatocellular carcinoma (HCC). miR-128-3p was recently reported to be deregulated in several types of cancer. However, the biological function and potential mechanisms of miR-128-3p in HCC remain unknown. In the present study, we found that miR-128-3p was frequently downregulated in HCC tissues and cell lines by qRT-PCR analysis. Moreover, functional assays showed that overexpression of miR-128-3p markedly suppressed HCC cell proliferation by inducing G1 phase cell arrest and migration. Mechanistically, miR-128-3p was confirmed to regulate PIK3R1 ( $\mathrm{p} 85 \alpha)$ expression thereby suppressing phosphatidylinositol 3-kinase (PI3K)/AKT pathway activation using qRT-PCR and western blot analysis. Furthermore, correlation analysis and Kaplan-Meier estimates revealed an inverse correlation between miR-128-3p and p85 $\alpha$ as well as a shorter disease-free survival (DFS) period after HCC resection in patients with low miR-128-3p expression. Hence, we conclude that miR-128-3p, which is frequently downregulated in $\mathrm{HCC}$,
\end{abstract}

Correspondence to: Professor Guo-Bin Wu or Professor Yin-Nong Zhao, Department of Hepatobiliary Surgery, Affiliated Tumor Hospital of Guangxi Medical University, 71 Hedi Road, Nanning, Guangxi 530021, P.R. China

E-mail: gb.wu@139.com

E-mail: hxphcy@126.com

*Contributed equally

Abbreviations: TGF- $\beta$, transforming growth factor- $\beta$; 3'-UTR, 3'-untranslated region; HCC, hepatocellular carcinoma; DFS, disease-free survival; PI3K, phosphatidylinositol 3-kinase; mTOR, mammalian target of rapamycin; FAK, focal adhesion kinase; ABCC5, ATP-binding cassette, sub-family C (CFTR/MRP), member 5; DCX, doublecortin

Key words: HCC, miR-128-3p, PIK3R1, proliferation, prognosis inhibits HCC progression by regulating PIK3R1 and PI3K/ $\mathrm{AKT}$ activation, and is a prognostic marker for $\mathrm{HCC}$ patients.

\section{Introduction}

Hepatocellular carcinoma (HCC) is the fifth most common type of cancer worldwide and the third leading cause of cancer-related deaths, with more than 700,000 cases being diagnosed yearly (1-3). The diagnosis and management of HCC have changed greatly within the past decade, but postoperative recurrence occurs frequently and the 5-year survival rate of HCC patients remains quite low $(2,4)$. Hepatocarcinogenesis is a complex and multistep process in which many signalling factors are altered, leading to a multifarious molecular profile (5-8). Although much effort has been made to identify key molecules involved in the development and progression of HCC, our understanding of the molecular pathogenesis of this disease remains elusive. Hence, there is an urgent need to develop novel strategies for the diagnosis, treatment and prognosis of HCC.

microRNAs (miRNAs) are endogenous, 22-nucleotide long, non-coding RNAs that negatively regulate the expression of multiple target genes at the post-transcriptional level by binding to the 3'-untranslated region (3'-UTR) of target mRNAs, resulting in mRNA degradation or blockade of mRNA translation (9). Growing evidence indicates that miRNAs play an important role in diverse biological processes, and aberrant expression of specific miRNAs is involved in a wide range of human cancers, functioning as classical oncogenes or tumor-suppressor genes $(10,11)$. Deregulation of miRNAs which have been associated with HCC patient clinicopathological features, can also contribute to HCC development by influencing cell growth, apoptosis, migration or invasion (12-15). Hence, more extensive investigations are needed to identify miRNAs in order to reveal the underlying mechanisms of HCC carcinogenesis and progression, and to facilitate targeted therapy and improve the prognosis of HCC patients.

Currently, gene expression profiling is employed to investigate the spectrum of differentially expressed genes in HCC cells and clinical specimens. Numerous candidate genes 
potentially involved in HCC developmental processes, such as proliferation, apoptosis, angiogenesis and invasion have been identified. Our previous microarray profiling showed that miR-128-3p is downregulated in HCC tissues. The role of miR-128-3p in HCC carcinogenesis and progression, however, remains unknown. In the present study, the roles of miR-128-3p in HCC development were investigated. We found that low miR-128-3p expression in HCC tissues was correlated with a worse prognosis for HCC patients. Additionally, we also found that miR-128-3p downregulated PIK3R1 to inhibit the PI3K-AKT pathway and thereby suppress HCC progression. Therefore, these findings demonstrate that miR-128-3p is a prognostic predictor for HCC patients, and provide new insights for the study of the molecular mechanisms of HCC and subsequent treatment.

\section{Materials and methods}

Patients and tissue samples. Surgically resected paired $\mathrm{HCC}$ and adjacent noncancerous tissues were collected from 72 primary HCC patients at The Affiliated Tumor Hospital of Guangxi Medical University between March 2011 and May 2013. Tissue samples were immediately frozen in liquid nitrogen until analysis. The cases selected were based on a clear pathological diagnosis, follow-up data, and had first undergone radical resection of $\mathrm{HCC}$, and had not received preoperative adjuvant chemotherapy, radiotherapy, targeted therapy or immunotherapy. Informed consent was obtained from each patient, and the study was approved by the Ethics Committee of Guangxi Medical University, Nanning, China. The investigations were conducted according to the Declaration of Helsinki Principles.

RNA extraction and quantitative RT-PCR. Total RNA, including miRNA, was extracted using TRIzol reagent (Invitrogen, Carlsbad, CA, USA) according to the manufacturer's instructions. cDNAs were synthesized using ReverTra Ace qPCR RT kit (FSQ-101; Toyobo, Kagoshima, Japan). microRNA was reversely transcribed using First Strand cDNA Synthesis kit ReverTra Ace - $\alpha$ - (FSK-100; Toyobo). Real-time PCR analyses were performed with Thunderbird SYBR qPCR mix (QPS-201; Toyobo) on an MxPro Mx3000P Sequence Detection system (Stratagene, La Jolla, CA, USA). U6 small nuclear RNA or $\beta$-actin was used as an internal normalized reference, and fold changes were calculated by relative quantification $\left(2^{-\Delta \Delta \mathrm{Ct}}\right)$. The primers used were: miR-128-3p specific stem-loop reverse transcription primers, 5'-GTCGTATCCAGTGCAGGGTCCGAGGTATTCGCACT GGATACGACAAAGAG-3'; miR-128-3p forward, 5'-GGTC ACAGTGAACCGGTC-3' and reverse, 5'-GTGCAGGGTCC GAGGT-3'; U6 forward, 5'-CTCGCTTCGGCAGCACA-3' and reverse, 5'-AACGCTTCACGAATTTGCGT-3'; PIK3R1 forward, 5'-AAGAAGTTGAACGAGTGGTTGG-3' and reverse, 5'-GCCCTGTTTACTGCTCTCCC-3'; $\beta$-actin forward, 5'-AGTGTGACGTTGACATCCGT-3' and reverse, 5'-GCAGCTCAGTAACAGTCCGC-3'. All samples were amplified in triplicate.

Cell culture and transfection. Cells were obtained from the Institute of Biochemistry and Cell Biology of the Chinese
Academy of Sciences (Shanghai, China). Human HCC cell lines (QGY-7703, SK-hep1, QGY-7404, SMMC-7721, Huh7 and HepG2) and human normal liver cells (HL-7702) were maintained in RPMI-1640 medium with $10 \%$ fetal bovine serum (FBS; Gibco, USA) at $37^{\circ} \mathrm{C}$ in a humidified incubator containing $5 \% \mathrm{CO}_{2}$. miR-128-3p duplex mimics and a negative control (NC) were obtained from Genepharma (Shanghai, China). Cells were transfected with RNAs using INTERFERin Transfection reagent (Polyplus Transfection, Illkirch, France) at a final concentration of $100 \mathrm{nM}$ according to the manufacturer's instructions.

Cell proliferation and colony formation assays. Cells were seeded into 96 -well plates $\left(5 \times 10^{3} /\right.$ well $)$ and transfected with miR-128-3p mimics or the NC. The cell proliferation of HCC cell lines was determined using WST-8 staining with the Cell Counting Kit-8 (Dojindo, Japan) at the indicated time-points (24, 48, 72 and $96 \mathrm{~h})$ according to the manufacturer's instructions. For the colony formation assay, cells were seeded into 6 -well plates at a low density $\left(1 \times 10^{3}\right.$ cells/well) and cultured for 10 days. Then, cells were fixed with $4 \%$ paraformaldehyde for $30 \mathrm{~min}$ and surviving colonies ( $>50$ cells/colony) were counted after staining with $1 \%$ crystal violet. The experiments were carried out in triplicate wells for at least 3 times.

Cell cycle distribution. Forty-eight hours after transfection in 6-well plates, the QGY-7703 or SK-hep1 cells were harvested and washed with cold $1 \mathrm{X}$ PBS. Then, cells were fixed in $70 \%$ ethanol at $4^{\circ} \mathrm{C}$ overnight, and washed with PBS twice, resuspended with $100 \mu \mathrm{l}$ RNase $\mathrm{A}$, and incubated at $37^{\circ} \mathrm{C}$ for $30 \mathrm{~min}$. Staining for DNA content was performed using $400 \mu \mathrm{l}$ propidium iodide (KeyGen, Nanjing, China) at $4^{\circ} \mathrm{C}$ for $30 \mathrm{~min}$ in the dark, and analyzed using an Epics XL flow cytometer (Beckman Coulter, Brea, CA, USA).

In vitro migration assay. Migration assays were performed using the 24-Well Cell Migration assay with an $8-\mu \mathrm{m}$ pore size polycarbonate membrane (Corning, New York, NY, USA), according to the manufacturer's instructions. Briefly, $24 \mathrm{~h}$ after the transfection, $5 \times 10^{4}$ QGY-7703 cells or 1x104 SK-hep1 cells were resuspended in $200 \mu \mathrm{l}$ serum-free medium and plated in the top chamber. The lower chambers were filled with $0.6 \mathrm{ml}$ of medium containing $10 \%$ FBS. Medium with $10 \%$ FBS was added to the lower chamber as a chemoattractant. After a 24-h incubation at $37^{\circ} \mathrm{C}$, the cells on the upper surface of the membrane were removed, and the cells on the lower surface were fixed, stained, photographed, and counted under a microscope in five fields.

Western blot analysis. Antibodies for p85, p-AKT (Ser473), p-mTOR, p-p70S6K and $\beta$-actin were purchased from Cell Signaling Technology, and all the antibodies were rabbit anti-human. Cells were harvested and then lysed with RIPA buffer supplemented with $1 \mathrm{mmol} / \mathrm{l}$ PMSF (both from Boster, Wuhan, China), and then centrifuged at 15,000 rpm at $4^{\circ} \mathrm{C}$ for $10 \mathrm{~min}$. Protein concentrations of the extracts were measured using the bicinchoninic acid (BCA) protein assay kit (KeyGen). Equal amounts of the proteins were concentrated and separated through SDS-PAGE, and then transferred to polyvinylidene difluoride (PVDF) membranes (Boster). After 
blocking in TBST (Tris-buffered saline with Tween-20) which contained 5\% non-fat milk for $60 \mathrm{~min}$, the membranes were incubated with the primary antibody $(1: 1,000$ dilution; $\beta$-actin, as a loading control, 1:2,500 dilution) overnight at $4^{\circ} \mathrm{C}$. The membranes were incubated with the secondary antibodies (mouse anti-rabbit and HRP-linked antibody, 1:5,000 dilution; Cell Signaling Technology). After incubating in enhanced chemiluminescence solution (Boster), the proteins on the membranes were detected using Bio-Rad Universal Hood III, and analyzed by Image $\mathrm{Lab}^{\mathrm{TM}}$ software 2.0 (Bio-Rad).

miRNA target predictions. Predicted targets of miR-128-3p and its sites were analyzed using TargetScan (http://www.targetscan.org/).

Statistical analysis. Data are presented as the mean \pm standard deviation (SD) of one representative experiment. The $\chi^{2}$ test and Fisher's exact test was used to analyze the relationship between the expression level of miR-128-3p and the clinicopathological characteristics. Unless otherwise noted, the differences between the groups were analyzed by one-way analysis of variance (ANOVA) when there were more than two groups. Differences in miR-128-3p expression between the HCC and noncancerous tissues of the human subjects were calculated using a two-tailed independent sample Student's t-test. Disease-free survival (DFS) was displayed by KaplanMeier survival curves, and DFS of the different groups was compared by log-rank test. The relationship between the expression level of miR-128-3p and P85 was measured by Pearson's correlation coefficient analysis. In all cases, differences were considered statistically significant at $\mathrm{P}<0.05$. All analyses were performed using SPSS 17.0 software (SPSS, Inc., Chicago, IL, USA).

\section{Results}

miR-128-3p is downregulated in the HCC tissues as well as in the HCC cell lines. In order to investigate the expression of miR-128-3p in HCC, the levels of miR-128-3p in 72 paired HCC tissues and $6 \mathrm{HCC}$ cell lines and human normal liver cells were tested by qRT-PCR. As shown in Fig. 1A, miR-128-3p expression was significantly downregulated in $65.30 \%$ (47 of 72) of the HCC samples compared to their matched controls. The median miR-128-3p expression level in all the HCC tissues was 22.5 -fold lower than that in the matched controls $(\mathrm{P}<0.001)$. In addition, the expression of miR-128-3p in the HCC cell lines (including SK-hep1, QGY-7404, SMMC7721, Huh7, QGY-7703, HepG2) was lower than that in the human normal liver cell line HL-7702 (Fig. 1B). These findings indicate that miR-128-3p was consistently decreased in HCC, which may contribute to HCC pathogenesis.

Correlation between miR-128-3p expression and the clinicopathological features of the HCC patients. To further understand the relationship between the miR-128-3p expression levels and clinicopathological factors, $72 \mathrm{HCC}$ patients who underwent radical resection and relapsed after a 3-year follow-up, were divided into a high or low miR-128-3p expression group according to the 50th percentile (median) of relative miR-128-3p expression, as analyzed by the $\chi^{2}$ test (Table I).
A

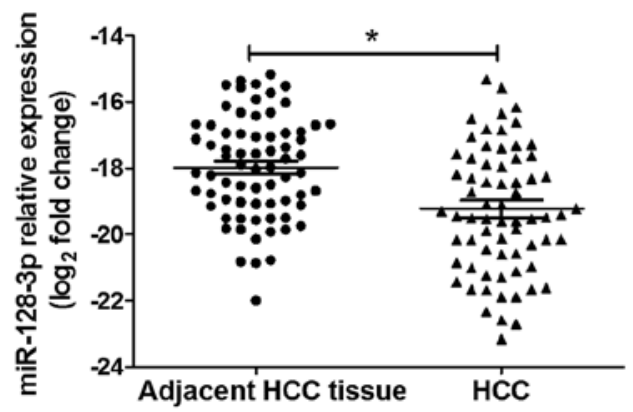

B

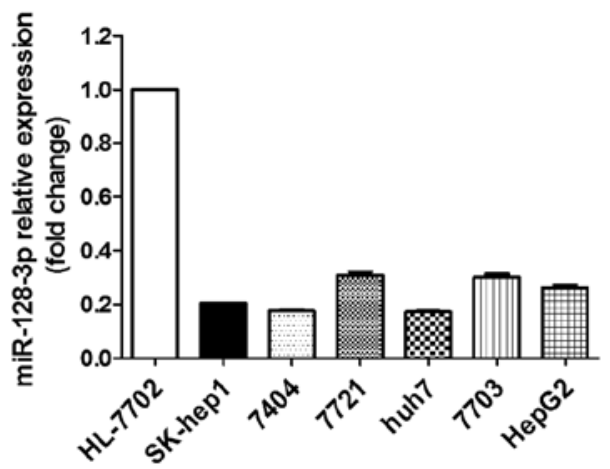

C

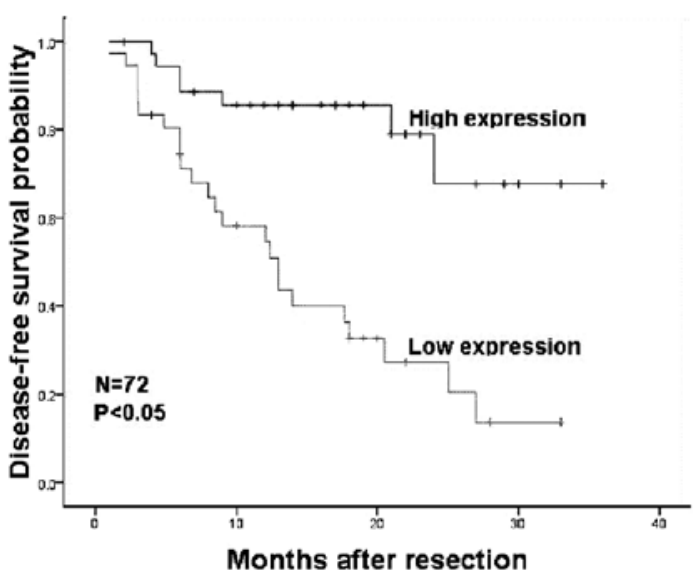

Figure 1. Levels of miR-128-3p expression in the HCC tissues and cell lines and its prognostic value in HCC patients. (A) miR-128-3p expression levels in 72 paired $\mathrm{HCC}$ tissues and their matched controls were analyzed by qRT-PCR. (B) miR-128-3p expression in HCC cells. (C) Patients with low miR-128-3p expression had poor DFS. Kaplan-Meier analyses of survival time in $72 \mathrm{HCC}$ patients according to the expression level of miR-128-3p. ${ }^{*} \mathrm{P}<0.05$. HCC, hepatocellular carcinoma; DFS, disease-free survival.

We found that low expression of miR-128-3p was strongly correlated with tumor-node metastasis (TNM) and tumor size $(\mathrm{P}<0.05)$. To exclude the confounder effect, we further performed Kaplan-Meier survival analysis and Cox proportional hazards regression analysis. Strikingly, the Kaplan-Meier survival analysis showed that low miR-128-3p expression was correlated with a shorter DFS $(\mathrm{P}<0.05)($ Fig. $1 \mathrm{C})$ in the HCC patients. Multivariate analysis further confirmed that a reduced miR-128-3p level is an independent predictor for a short DFS of HCC patients $(\mathrm{P}<0.05)$ (Table II). These results indicate that miR-128-3p may be involved in the tumor development and progression of HCC. 
Table I. Associations between the expression of miR-128-3p and the clinicopathological features of the HCC patients.

\begin{tabular}{|c|c|c|c|c|c|}
\hline \multirow[b]{2}{*}{ Characteristics } & \multirow[b]{2}{*}{ No. of patients } & \multicolumn{2}{|c|}{ miR-128-3p } & \multirow[b]{2}{*}{ P-value } & \multirow[b]{2}{*}{$\chi^{2}$} \\
\hline & & High, n (\%) & Low, n (\%) & & \\
\hline & 72 & $36(50.0)$ & $36(50.0)$ & & \\
\hline \multicolumn{6}{|l|}{ Gender } \\
\hline Male & 66 & $32(48.5)$ & $34(51.5)$ & 0.674 & 0.727 \\
\hline Female & 6 & $4(66.7)$ & $2(33.3)$ & & \\
\hline \multicolumn{6}{|l|}{ Age (years) } \\
\hline$\geq 50$ & 25 & $14(56.0)$ & $11(44.0)$ & 0.311 & 0.551 \\
\hline$<50$ & 47 & $22(46.8)$ & $25(53.2)$ & & \\
\hline \multicolumn{6}{|l|}{ Hepatitis B } \\
\hline Positive (+) & 64 & $32(55.6)$ & $32(44.4)$ & 1.000 & 0.000 \\
\hline Negative (-) & 8 & $4(49.2)$ & $4(50.8)$ & & \\
\hline \multicolumn{6}{|l|}{ Tumor size $(\mathrm{cm})$} \\
\hline$<5$ & 29 & $20(69.0)$ & $9(31.0)$ & 0.008 & 6.986 \\
\hline$\geq 5$ & 43 & $16(37.2)$ & $27(62.8)$ & & \\
\hline \multicolumn{6}{|l|}{ Tumor number } \\
\hline Solitary & 59 & $30(50.8)$ & $29(49.2)$ & 1.000 & 0.094 \\
\hline Multiple & 13 & $6(46.2)$ & $7(53.8)$ & & \\
\hline \multicolumn{6}{|l|}{ Tumor capsule } \\
\hline Void or particle & 31 & $17(54.8)$ & $14(45.2)$ & 0.634 & 0.510 \\
\hline Intact & 41 & $19(46.3)$ & $22(53.7)$ & & \\
\hline \multicolumn{6}{|l|}{$\operatorname{AFP}(n g / m l)$} \\
\hline$\leq 20$ & 21 & $13(62.5)$ & $8(37.5)$ & 0.150 & 1.681 \\
\hline$>20$ & 51 & $23(43.8)$ & $28(56.3)$ & & \\
\hline \multicolumn{6}{|l|}{ TNM } \\
\hline $\mathrm{I}+\mathrm{II}$ & 56 & $32(57.1)$ & $24(42.9)$ & 0.045 & 5.143 \\
\hline $\mathrm{III}+\mathrm{IV}$ & 16 & $4(25.0)$ & $12(75.0)$ & & \\
\hline
\end{tabular}

HCC, hepatocellular carcinoma; TNM, tumor-node metastasis; AFP, $\alpha$-fetoprotein.

Table II. Multivariate Cox regression analyses of overall survival in the 72 patients with HCC.

\begin{tabular}{lll}
\hline Tumor characteristics & Relative risk $(95 \% \mathrm{CI})$ & P-value \\
\hline Tumor size $(>5 \mathrm{~cm})$ & $0.259(0.046-1.440)$ & 0.123 \\
Age (years) & $1.442(0.590-3.521)$ & 0.422 \\
Tumor number & $0.580(0.146-2.301)$ & 0.439 \\
Tumor capsule & $0.747(0.316-1.766)$ & 0.507 \\
Hepatitis B & $3.387(0.447-25.676)$ & 0.238 \\
AFP (>20 ng/ml) & $0.757(0.282-2.029)$ & 0.579 \\
TNM (III+IV) & $0.620(0.000-9.632 \mathrm{E} 72)$ & 0.996 \\
miR-128-3p (low) & $0.323(0.121-0.864)$ & 0.024
\end{tabular}

HCC, hepatocellular carcinoma; CI, confidence interval; TNM, tumor-node metastasis; AFP, $\alpha$-fetoprotein.

miR-128-3p overexpression suppresses HCC cell proliferation and clonogenicity. Following the finding of decreased expression of miR-128-3p in HCC, it was then determined whether
miR-128-3p functions as a tumor suppressor. The effect of miR-128-3p on HCC cell growth was observed. Firstly, two of the HCC cell lines were randomized to do follow-up experiments. The transfection efficiency of miR-128-3p mimics in the SK-hep1 and QGY-7703 HCC cells was assessed by qRT-PCR after transfection with the miR-128-3p mimics and NC after $24 \mathrm{~h}$. As shown in Fig. 2A, the expression of miR-128-3p in the HCC cells was significantly increased after transfection with the miRNA mimics. Then, we evaluated the effect of miR-128-3p overexpression on the proliferation of the HCC cells. The results showed that proliferation of the HCC cells was suppressed by miR-128-3p overexpression (Fig. 2B). We also further investigated the effect of miR-128-3p overexpression on the clonogenicity of the SK-hep1 and QGY-7703 cells, which were transfected with the miR-128-3p mimics or the NC. Compared with the NC transfectants, HCC cells transfected with the miR-128-3p mimics displayed notably fewer colonies (Fig. 2C).

miR-128-3p inhibits HCC cell cycle progression to suppress tumor growth. Since overexpression of miR-128-3p inhibited HCC cell proliferation, we ascertained whether the effect of 

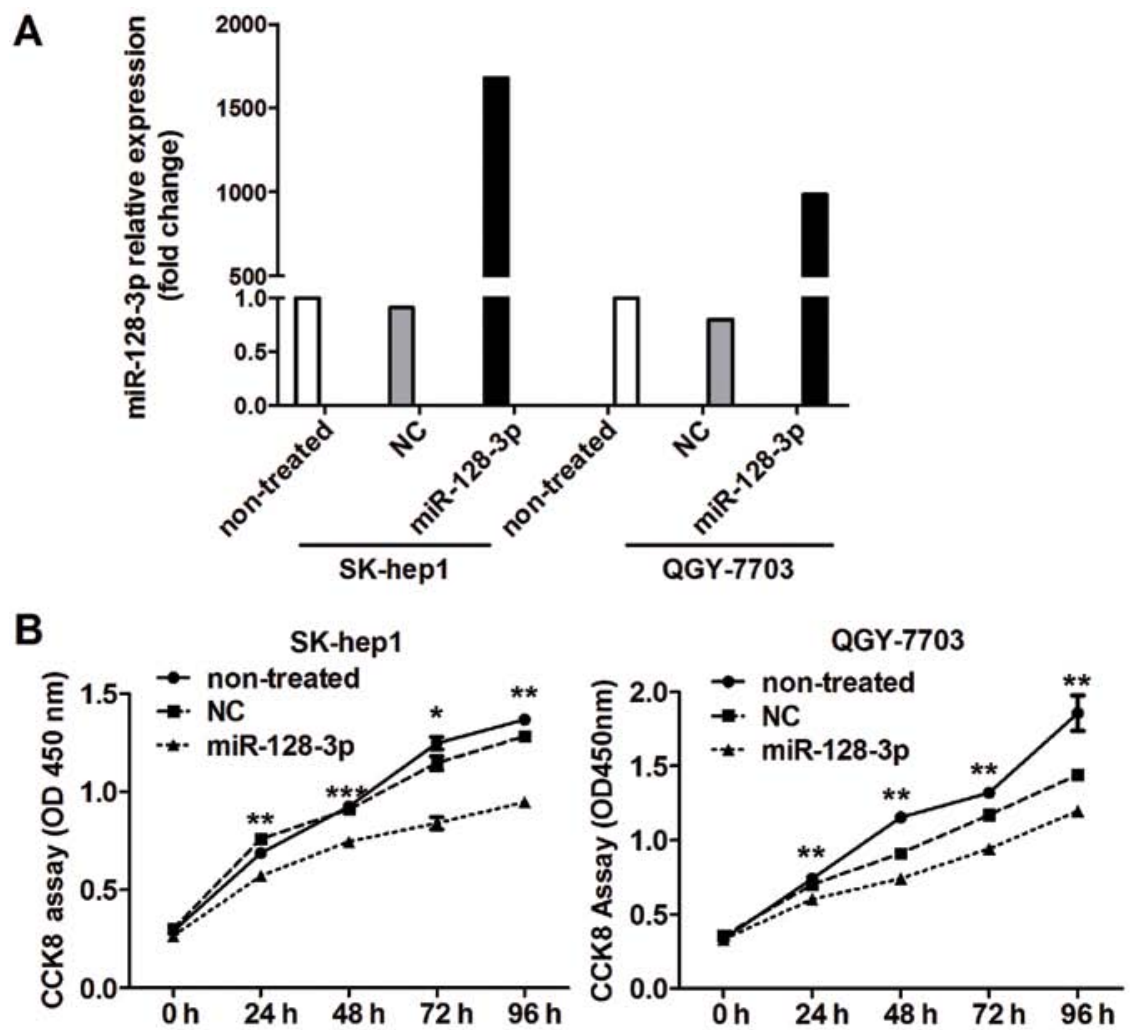

C
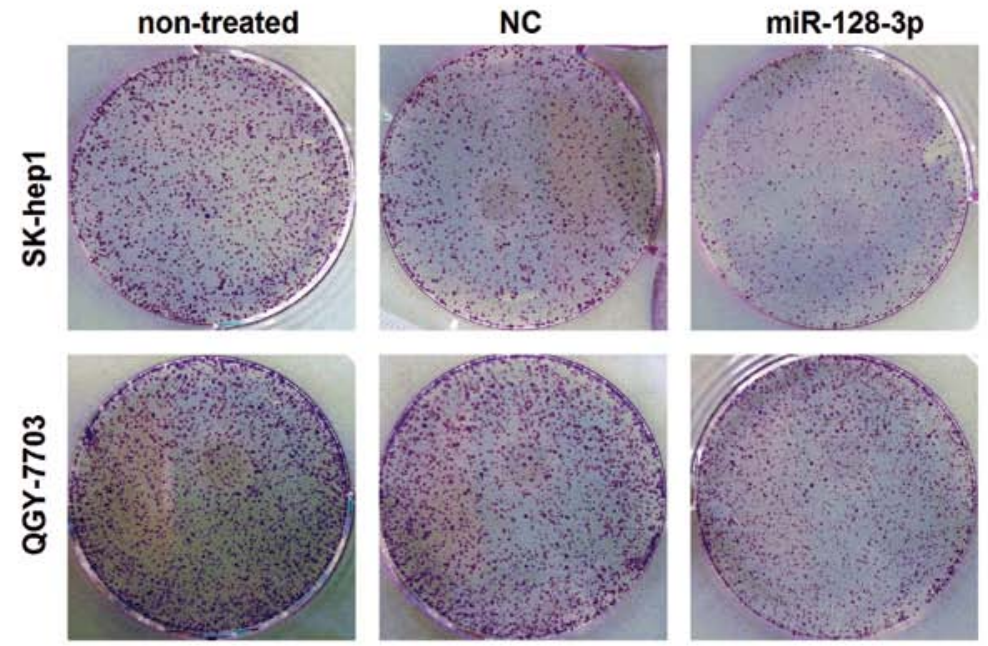

Figure 2. Overexpression of miR-128-3p suppresses HCC growth in vitro. (A) miR-128-3p relative expression in the SK-hep1 and QGY-7703 cells after transfection with the miR-128-3p mimics or the NC duplex for $24 \mathrm{~h}$. (B) Cell proliferation of the HCC cell lines was evaluated using CCK-8 assay after transfection with miR-128-3p or NC at the indicated times. (C) Effect of miR-128-3p on the colony formation of the HCC cell lines. Data are shown as the mean \pm SD ( $\mathrm{n}=4$ ) of one representative experiment. Similar results were obtained in three independent experiments. ${ }^{*} \mathrm{P}<0.05,{ }^{* *} \mathrm{P}<0.01,{ }^{* * * *} \mathrm{P}<0.001$.

miR-128-3p is relevant to the cell cycle. Cell cycle analysis of the HCC SK-hep1 and QGY-7703 cell lines indicated that miR-128-3p inhibited cell cycle progression, most likely due to G0-G1 phase arrest $(\mathrm{P}<0.05)($ Fig. 3). These results indicate that miR-128-3p can inhibit HCC cell growth through inhibition of cell cycle progression.

miR-128-3p inhibits HCC cell migration. The role of miR-128-3p in HCC cell migration was then investigated. As shown in Fig. 4, HCC SK-hep-1 and QGY-7703 cells transfected with the miR-128-3p mimics had significantly weaker migratory ability when compared to that of the control cells.
These observations imply that miR-128-3p may inhibit HCC metastasis.

miR-128-3p inhibits PI3K/AKT pathway activation by downregulating $p 85 \alpha$ expression. To elucidate the underlying molecular mechanisms of miR-128-3p in proliferation and migration, 1,047 putatively conserved gene targets of miR-128-3p in TargetScan (http://www.targetscan.org) were subjected to enrichment analysis of the cell signaling pathways using the Kyoto Encyclopedia of Genes and Genomes (KEGG) pathway database (http://www.genome.jp/kegg/). It was found that the signaling pathway in cancer (map05200) 
A

non-treated

NC

$\operatorname{miR}-128-3 p$
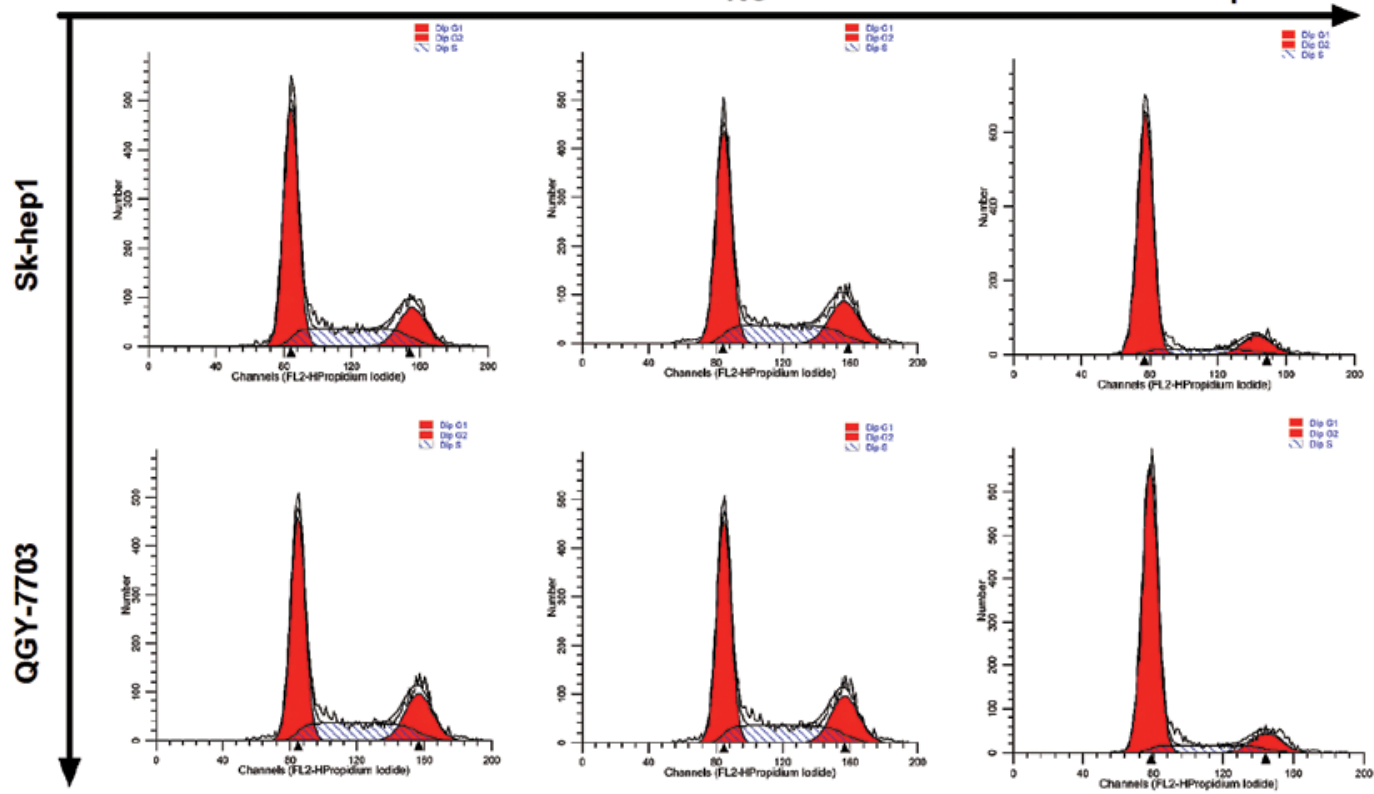

B

SK-hep1
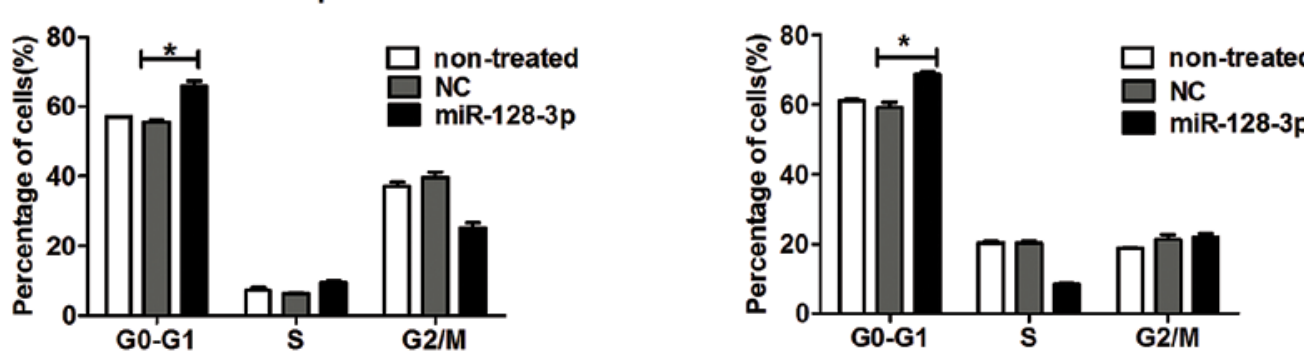

Figure 3. Overexpression of miR-128-3p inhibits HCC proliferation by inhibiting cell cycle progression. (A) HCC SK-hep1 and QGY-7703 cells, which were transfected with miR-128-3p or NC for $48 \mathrm{~h}$, were harvested and analyzed by FACS and (B) the cell cycle distribution was determined. Data are shown as mean $\pm \mathrm{SD}(\mathrm{n}=4)$ of one representative experiment. Similar results were obtained in three independent experiments. " $\mathrm{P}<0.05$. HCC, hepatocellular carcinoma.
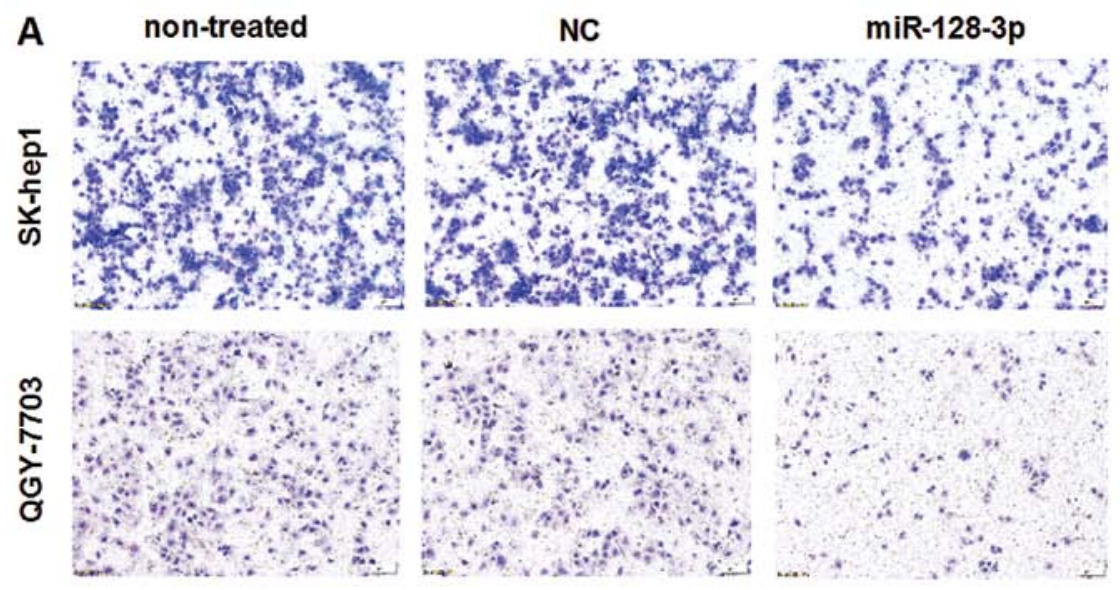

B

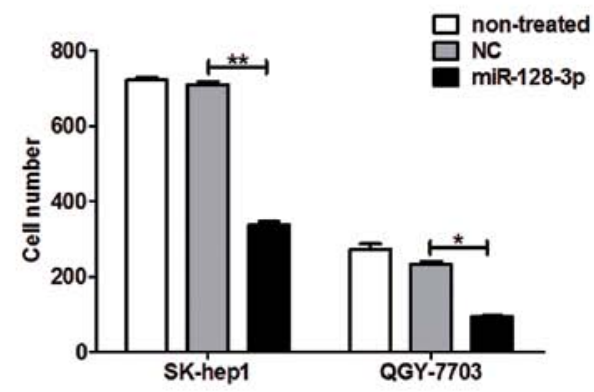

Figure 4. miR-128-3p inhibits cell migration in HCC cells. (A) Transwell migration assays in the SK-hep1 and QGY-7703 cells transfected with miR-128-3p or NC. (B) The number of cells was calculated with crystal violet staining. Data are shown as mean \pm SD $(n=4)$ of one representative experiment. Similar results were obtained in three independent experiments. ${ }^{*} \mathrm{P}<0.05,{ }^{* * *} \mathrm{P}<0.01$. HCC, hepatocellular carcinoma; NC, negative control.

and PI3K/AKT (Table III) were the most significantly enriched pathways compared to the other signaling pathways. As shown in Fig. 5A, the human PIK3R1, encoding p85 $\alpha$ and involving $28.77 \%$ of the 212 miR-128-3p-related pathways, is a member of the pathway in cancer and the PI3K-AKT signaling pathway, which is known to be involved in cancer 
A

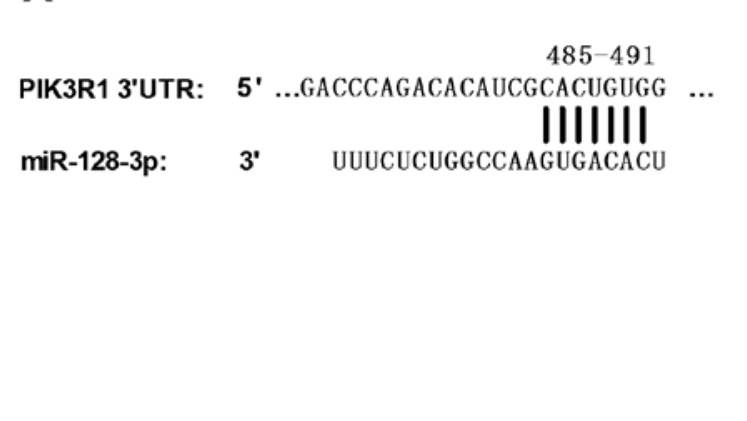

B

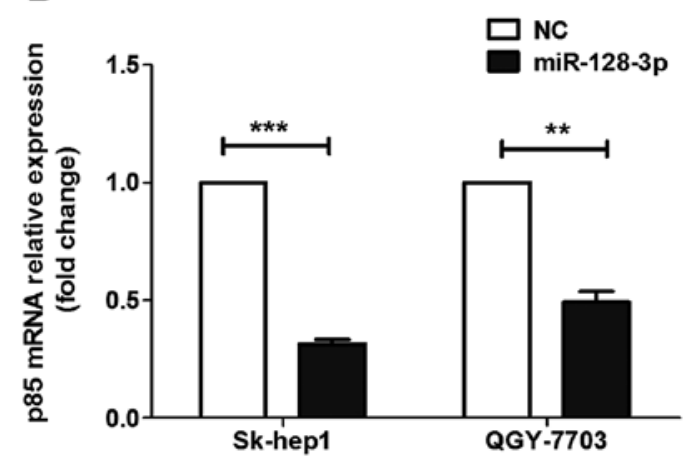

C

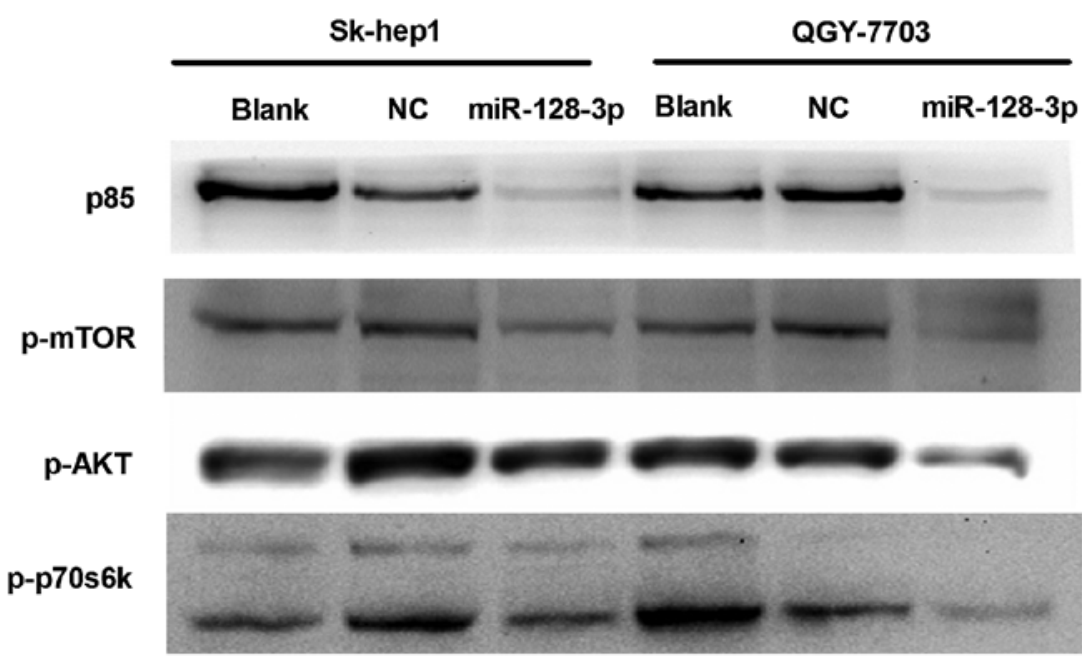

$\beta$-actin

D
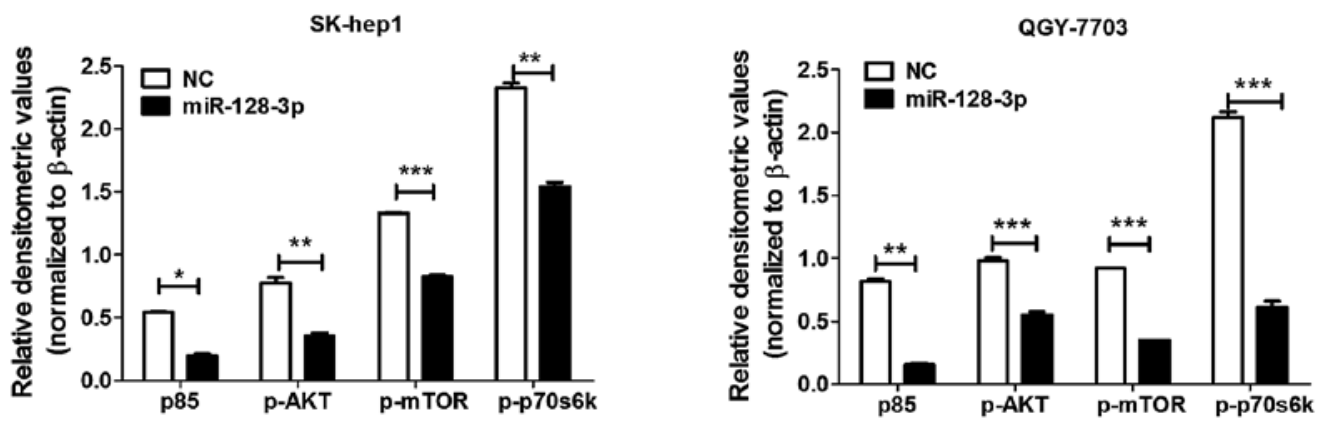

Figure 5. miR-128-3p targets p85 $\alpha$ and inhibits PI3K-AKT pathway activation. (A) Sequence alignment of miR-128-3p and its conserved target site in p85a 3'UTR. (B) p85 $\alpha$ mRNA in the SK-hep1 and QGY-7703 cells was analyzed by qRT-PCR after transfection of miR-128-3p or NC for $48 \mathrm{~h}$. (C and D) Protein levels of the indicated PI3K-AKT pathway members were analyzed in the SK-hep1 and QGY-7703 cells after transfection with miR-128-3p or NC for 48 h. Data are shown as mean $\pm \mathrm{SD}(\mathrm{n}=4)$ of one representative experiment. Representative images of one experiment are shown. Similar results were obtained in three independent experiments. ${ }^{*} \mathrm{P}<0.05,{ }^{* *} \mathrm{P}<0.01,{ }^{* * *} \mathrm{P}<0.001$. HCC, hepatocellular carcinoma; NC, negative control.

development (16-19), and has one miR-128-3p binding site in its 3'-UTR (Fig. 5A). Therefore, to verify whether miR-128-3p regulates PIK3R1, the cellular mRNA expression of $\mathrm{p} 85 \alpha$ was detected by qRT-PCR after treatment with the miRNA mimics for $24 \mathrm{~h}$. Compared with the NC duplex, the p85 mRNA expression in the HCC cells was extremely suppressed by the miR-128-3p mimics (Fig. 5B). In addition, p85 $\alpha$ was found to be related to the activation of the PI3K/AKT pathway. Therefore, whether miR-128-3p influences the activation of the PI3K pathway was also studied. Phosphorylation of the essen- tial molecules in the PI3K pathway was analyzed by western blot analysis. As shown in Fig. 5C and D, the protein levels of p85 $\alpha$, phosphorylated AKT, mammalian target of rapamycin (p-mTOR) and p-p70S6K were inhibited by miR-128-3p overexpression in the HCC SK-hep1 and QGY-7703 cells.

To confirm the relevance of our in vitro findings, $\mathrm{p} 85 \alpha$ expression was assessed in the same 72 HCC samples. As shown in Fig. 6, p85 $\alpha$ expression was upregulated in $68.06 \%$ (49 of 72) of the HCC samples and showed an inverse correlation with miR-128-3p expression in the HCC samples. 
Table III. Enrichment analysis of predicted miR-128-3p targets in the KEGG signaling pathway database.

\begin{tabular}{lc}
\hline Pathway & Count \\
\hline Pathways in cancer & 32 \\
PI3K-Akt signaling pathways & 30 \\
MAPK signaling pathways & 29 \\
Proteoglycans in cancer & 26 \\
Rap1 signaling pathway & 25 \\
Ras signaling pathway & 24 \\
Transcriptional misregulation in cancer & 23 \\
Focal adhesion kinase & 21
\end{tabular}

Only the top 8 enriched cell signaling pathways of predicted miR-128-3p targets in KEGG database are shown. HCC, hepatocellular carcinoma; KEGG, Kyoto Encyclopedia of Genes and Genomes.
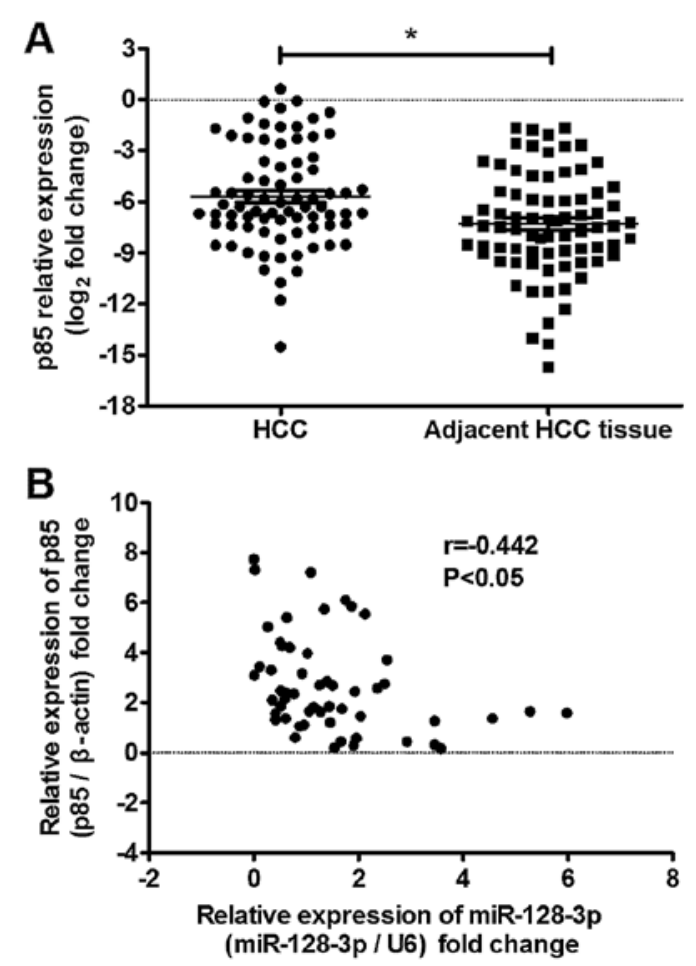

Figure 6. Expression of $\mathrm{p} 85 \alpha$ is frequently upregulated in HCC and is inversely related to miR-128-3p expression. (A) The expression level of $\mathrm{p} 85 \alpha$ in the $72 \mathrm{HCC}$ samples was tested by qPCR. (B) miR-128-3p and p $85 \alpha$ were inversely expressed in the 72 HCC samples. ${ }^{*} \mathrm{P}<0.05$. HCC, hepatocellular carcinoma.

These findings further suggest that decreased expression of miR-128-3p triggered upregulation of p85 $\alpha$ partially in HCC, and demonstrate that miR-128-3p can inhibit HCC progression by downregulating PIK3R1 expression and repressing PI3K-AKT pathway activation.

\section{Discussion}

In recent years, growing evidence suggests that aberrant expression of miRNAs contributes to tumorigenesis. Changes in miRNA profiling are implicated in almost all aspects of cancer biology, including cell proliferation, apoptosis, migration and angiogenesis. Thus, miRNAs are increasingly viewed as potential diagnostic and therapeutic tools. In the present study, miR-128-3p was found to be markedly decreased in HCC. Low expression of miR-128-3p was significantly associated with a worse prognosis for HCC patients. Moreover, miR-128-3p may function as a tumor suppressor, as it was found to be involved in the development and progression of HCC through repressing PI3K/AKT pathway activation by regulating $\mathrm{p} 85 \alpha$. These results suggest that miR-128-3p may be a new prognostic predictor as well as a potential therapeutic target for HCC.

Concerning the roles of miR-128 in tumorigenesis and development, research has demonstrated that miR-128 can regulate proliferation, differentiation and apoptosis of various types of tumor cells. For example, P70S6k1 is known as one of the key downstream targets of mTOR and is involved in tumor angiogenesis. Shi et al (20) found that miR-128 overexpression acted as a tumor suppressor by targeting p70S6K1 consequently attenuating tumor growth and angiogenesis in glioma. miR-128 also suppressed prostate and breast cancer by inhibiting BIM-1 in tumor-initiating cells consequently influencing the self-renewal and malignant transformation of tumor stem cells $(21,22)$. Moreover, Zhu et al (23) found that reduced miR-128 induced chemotherapeutic resistance via influencing multidrug resistance associated protein (ABCC5, MRP5). Research indicated that miR-128 overexpression can inhibit Reelin and doublecortin (DCX) expression consequently reducing neuroblastoma cell motility and invasiveness (24). In the present study, overexpression of miR-128-3p in the HCC cells not only inhibited HCC proliferation by arresting the cell cycle at the G1 phase, but also suppressed HCC cell colony formation and migration. These results indicate that miR$128-3 \mathrm{p}$ can act as a tumor suppressor and is involved in the tumor development and progression of HCC.

Identifying the molecular markers correlated with the survival of cancer patients has attracted much research interest. Hence, the suppressor role of miR-128-3p motivated us to detect the relationship between the miR-128-3p expression levels and clinicopathological factors. Firstly, the expression of miR-128-3p was found to be markedly decreased in HCC. This result was similar to previous studies that miR$128-3 p$ is repressed in ovarian cancer, non-small cell lung cancer, glioma progression, and in acute myeloid leukemia cells (25-28). Conversely, miR-128 expression was reported to be high in acute leukemia, and in undifferentiated gastric and prostate cancer $(22,29,30)$. Thus, miR-128-3p is a tissuespecific gene. Furthermore, we analyzed the relationship between miR-128-3p expression and the clinicopathological features of HCC and found that low expression of miR-128-3p was strongly correlated with TNM and was correlated with a shorter DFS in the HCC patients. These results are consistent with previous reports $(22,31)$ that the level of miR-128-3p is an independent predictor for reduced DFS of HCC patients.

Before further discussing the antitumor molecular mechanisms of miR-128-3p in HCC, it is important to note that the mechanisms responsible for miR-128-3p downregulation in cancers are largely unknown. According to previous studies, there are several possible reasons for the downregulation of 
miR-128-3p in HCC tissues. Firstly, miR-128-3p (known as miR-128) is the same major mature microRNA of miR-128-1 and miR-128-2. miR-128-1 and miR-128-2 are located on chromosomes $2 q$ and $3 q$, respectively (26). This location contains multiple tumor-suppressor genes and is one which commonly presents with loss of heterozygosity in various types of tumors, such as HCC (32), clear cell renal carcinoma (33) and lung cancer (34). Allelic loss of the genomic region may be responsible for the downregulation of miR-128-3p. Secondly, epigenetic alteration through DNA methylation also causes miR-128-3p downregulation $(28,31,35)$. Additionally, the expression of miR-128 can be regulated by a transcriptional factor. Snail and p53 directly bind to the promoter region of miR-128 consequently influencing the expression of miR-128 (27,36,37). Further studies are required to evaluate the cause of miR-128-3p deregulation in HCC development.

To elucidate the antitumor mechanism of miR-128-3p in $\mathrm{HCC}$, the target genes of miR-128-3p were investigated. One such target gene was $\mathrm{p} 85 \alpha$, a well-accepted regulatory subunit of Class 1A PI3K. To investigate the role of p85 $\alpha$ in tumorigenesis, research on gain-of-function mutations in the $\mathrm{nSH}_{2}$ and/or iSH2 domain of p85 $\alpha$ have revealed that these mutations can relieve the repression on p110 catalytic activity and enhance PI3K signaling (38-41). Moreover, depletion of PI3K p85 $\alpha$ can decrease the expression of cyclin D1, CDK4 and p27/ kip1 and induce tumor cell apoptosis in colorectal cancer by negatively regulating the activity of Forkhead family transcription factors (42). Regarding metastasis, Hong et al (43) reported that the activation of non-smad TGF- $\beta$ signaling can promote mesenchymal transition depending upon focal adhesion kinase (FAK) binding with $\mathrm{p} 85 \alpha$. Hence, $\mathrm{p} 85$ can act as an oncogene in tumorigenesis. In the present study, p85 $\alpha$ was found to be upregulated in the HCC tissues, and correlation analysis showed an inverse correlation linking miR-128-3p and $\mathrm{p} 85 \alpha$ expression. Restoring miR-128-3p significantly repressed the PI3K/AKT pathway activation by downregulating $\mathrm{p} 85 \alpha$, thereby explaining why miR-128-3p suppresses HCC cell proliferation and metastasis.

It has been acknowledged that a single miRNA can regulate the expression of multiple genes by targeting different mRNAs (44), indicating that there may be other molecules or signaling pathways also targeted by miR-128-3p. This presumption requires future research to reveal the complete function of miR-128-3p in HCC carcinogenesis and progression.

In summary, we demonstrated that miR-128-3p is commonly downregulated in HCC, and is closely associated with the prognosis of HCC patients. miR-128-3p acts as a tumor-suppressor by silencing PI3KR1 to regulate the $\mathrm{PI} 3 \mathrm{~K} / \mathrm{AKT}$ signaling pathway. Further investigation is required to fully reveal the molecular mechanisms of miR-128-3p and to determine whether this miRNA is a potential therapeutic target for the treatment of HCC.

\section{Acknowledgements}

The present study was supported by grants from the Key Research Project of the Health Department of Guangxi Zhuang Autonomous Region (no. s201301-10), and the National Natural Science Foundation of China (no. 81360315).

\section{References}

1. Jemal A, Bray F, Center MM, Ferlay J, Ward E and Forman D: Global cancer statistics. CA Cancer J Clin 61: 69-90, 2011.

2. Forner A, Llovet JM and Bruix J: Hepatocellular carcinoma. Lancet 379: 1245-1255, 2012.

3. Ferlay J, Shin HR, Bray F, Forman D, Mathers C and Parkin DM: Estimates of worldwide burden of cancer in 2008: GLOBOCAN 2008. Int J Cancer 127: 2893-2917, 2010.

4. Bruix J, Gores GJ and Mazzaferro V: Hepatocellular carcinoma: Clinical frontiers and perspectives. Gut 63: 844-855, 2014.

5. Villanueva A, Newell P, Chiang DY, Friedman SL and Llovet JM: Genomics and signaling pathways in hepatocellular carcinoma. Semin Liver Dis 27: 55-76, 2007.

6. Aravalli RN, Cressman EN and Steer CJ: Cellular and molecular mechanisms of hepatocellular carcinoma: An update. Arch Toxicol 87: 227-247, 2013.

7. Moeini A, Cornellà $\mathrm{H}$ and Villanueva $\mathrm{A}$ : Emerging signaling pathways in hepatocellular carcinoma. Liver Cancer 1: 83-93, 2012.

8. Aravalli RN, Steer CJ and Cressman EN: Molecular mechanisms of hepatocellular carcinoma. Hepatology 48: 2047-2063, 2008.

9. Ambros V: The functions of animal microRNAs. Nature 431: $350-355,2004$

10. Ventura A and Jacks T: MicroRNAs and cancer: Short RNAs go a long way. Cell 136: 586-591, 2009.

11. Kong YW, Ferland-McCollough D, Jackson TJ and Bushell M: microRNAs in cancer management. Lancet Oncol 13: e249-e258, 2012.

12. Xu T, Zhu Y, Xiong Y, Ge YY, Yun JP and Zhuang SM: MicroRNA-195 suppresses tumorigenicity and regulates G1/S transition of human hepatocellular carcinoma cells. Hepatology 50: 113-121, 2009.

13. Hou J, Lin L, Zhou W, Wang Z, Ding G, Dong Q, Qin L, Wu X, Zheng Y, Yang Y, et al: Identification of miRNomes in human liver and hepatocellular carcinoma reveals miR-199a/b-3p as therapeutic target for hepatocellular carcinoma. Cancer Cell 19: 232-243, 2011.

14. Li D, Liu X, Lin L, Hou J, Li N, Wang C, Wang P, Zhang Q, Zhang P, Zhou W, et al: MicroRNA-99a inhibits hepatocellular carcinoma growth and correlates with prognosis of patients with hepatocellular carcinoma. J Biol Chem 286: 36677-36685, 2011.

15. Zhou X, Zhang CZ, Lu SX, Chen GG, Li LZ, Liu LL, Yi C, Fu J, $\mathrm{Hu} \mathrm{W}$, Wen JM, et al: miR-625 suppresses tumour migration and invasion by targeting IGF2BP1 in hepatocellular carcinoma. Oncogene 34: 965-977, 2015.

16. Gedaly R, Angulo P, Hundley J, Daily MF, Chen C, Koch A and Evers BM: PI-103 and sorafenib inhibit hepatocellular carcinoma cell proliferation by blocking Ras/Raf/MAPK and PI3K-AKT-mTOR pathways. Anticancer Res 30: 4951-4958, 2010.

17. Chen M, Gu J, Delclos GL, Killary AM, Fan Z, Hildebrandt MA, Chamberlain RM, Grossman HB, Dinney CP and Wu X: Genetic variations of the PI3K-AKT-mTOR pathway and clinical outcome in muscle invasive and metastatic bladder cancer patients. Carcinogenesis 31: 1387-1391, 2010.

18. Whittaker S, Marais R and Zhu AX: The role of signaling pathways in the development and treatment of hepatocellular carcinoma. Oncogene 29: 4989-5005, 2010.

19. Zhao L and Vogt PK: Class I PI3K in oncogenic cellular transformation. Oncogene 27: 5486-5496, 2008.

20. Shi ZM, Wang J, Yan Z, You YP, Li CY, Qian X, Yin Y, Zhao P, Wang YY, Wang XF, et al: MiR-128 inhibits tumor growth and angiogenesis by targeting p70S6K1. PLoS One 7: e32709, 2012.

21. Jin M, Zhang T, Liu C, Badeaux MA, Liu B, Liu R, Jeter C, Chen X, Vlassov AV and Tang DG: miRNA-128 suppresses prostate cancer by inhibiting BMI-1 to inhibit tumor-initiating cells. Cancer Res 74: 4183-4195, 2014.

22. Zhu YD, Wang L, Sun C, Fan L, Zhu DX, Fang C, Wang YH, Zou ZJ, Zhang SJ, Li JY, et al: Distinctive microRNA signature is associated with the diagnosis and prognosis of acute leukemia. Med Oncol 29: 2323-2331, 2012.

23. Zhu Y, Yu F, Jiao Y, Feng J, Tang W, Yao H, Gong C, Chen J, Su F, Zhang Y, et al: Reduced miR-128 in breast tumor-initiating cells induces chemotherapeutic resistance via Bmi-1 and ABCC5. Clin Cancer Res 17: 7105-7115, 2011.

24. Li M, Fu W, Wo L, Shu X, Liu F and Li C: miR-128 and its target genes in tumorigenesis and metastasis. Exp Cell Res 319: 3059-3064, 2013 
25. Li B, Chen $\mathrm{H}, \mathrm{Wu} \mathrm{N}$, Zhang WJ and Shang LX: Deregulation of miR-128 in ovarian cancer promotes cisplatin resistance. Int J Gynecol Cancer 24: 1381-1388, 2014.

26. Hu J, Cheng Y, Li Y, Jin Z, Pan Y, Liu G, Fu S, Zhang Y, Feng K and Feng Y: microRNA-128 plays a critical role in human non-small cell lung cancer tumourigenesis, angiogenesis and lymphangiogenesis by directly targeting vascular endothelial growth factor-C. Eur J Cancer 50: 2336-2350, 2014.

27. Dong Q, Cai N, Tao T, Zhang R, Yan W, Li R, Zhang J, Luo H, Shi Y, Luan W, et al: An axis involving SNAI1, microRNA-128 and SP1 modulates glioma progression. PLoS One 9: e98651, 2014.

28. Seca H, Lima RT, Almeida GM, Sobrinho-Simoes M, Bergantim R, Guimaraes JE and Vasconcelos MH: Effect of miR-128 in DNA damage of HL-60 acute myeloid leukemia cells. Curr Pharm Biotechnol 15: 492-502, 2014.

29. Khan AP, Poisson LM, Bhat VB, Fermin D, Zhao R, Kalyana-Sundaram S, Michailidis G, Nesvizhskii AI, Omenn GS, Chinnaiyan AM, et al: Quantitative proteomic profiling of prostate cancer reveals a role for miR-128 in prostate cancer. Mol Cell Proteomics 9: 298-312, 2010.

30. Katada T, Ishiguro H, Kuwabara Y, Kimura M, Mitui A, Mori Y, Ogawa R, Harata K and Fujii Y: microRNA expression profile in undifferentiated gastric cancer. Int J Oncol 34: 537-542, 2009.

31. Takahashi Y, Iwaya T, Sawada G, Kurashige J, Matsumura T, Uchi R, Ueo H, Takano Y, Eguchi H, Sudo T, et al: Up-regulation of NEK2 by microRNA-128 methylation is associated with poor prognosis in colorectal cancer. Ann Surg Oncol 21: 205-212, 2014.

32. Zhang X, Li HM, Liu Z, Zhou G, Zhang Q, Zhang T, Zhang J and Zhang C: Loss of heterozygosity and methylation of multiple tumor suppressor genes on chromosome 3 in hepatocellular carcinoma. J Gastroenterol 48: 132-143, 2013.

33. Gatto F, Nookaew I and Nielsen J: Chromosome 3p loss of heterozygosity is associated with a unique metabolic network in clear cell renal carcinoma. Proc Natl Acad Sci USA 111: E866-E875, 2014

34. Saint-Georges F, Garçon G, Escande F, Abbas I, Verdin A, Gosset P, Mulliez P and Shirali P: Role of air pollution Particulate Matter (PM(2.5)) in the occurrence of loss of heterozygosity in multiple critical regions of $3 \mathrm{p}$ chromosome in human epithelial lung cells (L132). Toxicol Lett 187: 172-179, 2009.
35. Mi S, Lu J, Sun M, Li Z, Zhang H, Neilly MB, Wang Y, Qian Z, Jin J, Zhang Y, et al: MicroRNA expression signatures accurately discriminate acute lymphoblastic leukemia from acute myeloid leukemia. Proc Natl Acad Sci USA 104: 19971-19976, 2007.

36. Tao T, Li G, Dong Q, Liu D, Liu C, Han D, Huang Y, Chen S, Xu B and Chen M: Loss of SNAIL inhibits cellular growth and metabolism through the miR-128-mediated RPS6KB1/HIF-1 $\alpha /$ PKM2 signaling pathway in prostate cancer cells. Tumour Biol 35: 8543-8550, 2014.

37. Adlakha YK and Saini N: miR-128 exerts pro-apoptotic effect in a p53 transcription-dependent and -independent manner via PUMA-Bak axis. Cell Death Dis 4: e542, 2013.

38. Cheung LW, Hennessy BT, Li J, Yu S, Myers AP, Djordjevic B, $\mathrm{Lu} \mathrm{Y,} \mathrm{Stemke-Hale} \mathrm{K,} \mathrm{Dyer} \mathrm{MD,} \mathrm{Zhang} \mathrm{F,} \mathrm{et} \mathrm{al:} \mathrm{High} \mathrm{frequency}$ of PIK3R1 and PIK3R2 mutations in endometrial cancer elucidates a novel mechanism for regulation of PTEN protein stability. Cancer Discov 1: 170-185, 2011.

39. Jaiswal BS, Janakiraman V, Kljavin NM, Chaudhuri S, Stern HM, Wang W, Kan Z, Dbouk HA, Peters BA, Waring P, et al: Somatic mutations in p85 $\alpha$ promote tumorigenesis through class IA PI3K activation. Cancer Cell 16: 463-474, 2009.

40. Sun M, Hillmann P, Hofmann BT, Hart JR and Vogt PK: Cancer-derived mutations in the regulatory subunit $\mathrm{p} 85 \alpha$ of phosphoinositide 3-kinase function through the catalytic subunit p110 $\alpha$. Proc Natl Acad Sci USA 107: 15547-15552, 2010.

41. Urick ME, Rudd ML, Godwin AK, Sgroi D, Merino M and Bell DW: PIK3R1 (p85 $\alpha$ ) is somatically mutated at high frequency in primary endometrial cancer. Cancer Res 71: 4061-4067, 2011.

42. Sun Y,Zhao S, Tian H, Xie X, Xiao F, Li K and Song Y: Depletion of PI3K p85 $\alpha$ induces cell cycle arrest and apoptosis in colorectal cancer cells. Oncol Rep 22: 1435-1441, 2009.

43. Hong M, Wilkes MC, Penheiter SG, Gupta SK, Edens M and Leof EB: Non-Smad transforming growth factor- $\beta$ signaling regulated by focal adhesion kinase binding the $\mathrm{p} 85$ subunit of phosphatidylinositol 3-kinase. J Biol Chem 286: 17841-17850, 2011.

44. Selbach M, Schwanhäusser B, Thierfelder N, Fang Z, Khanin R and Rajewsky N: Widespread changes in protein synthesis induced by microRNAs. Nature 455: 58-63, 2008. 